\title{
Effectiveness of Influenza Vaccines in the HIVE Household Cohort Over 8 Years: Is There Evidence of Indirect Protection?
}

Ryan E. Malosh, ${ }^{1}$ Joshua G. Petrie, ${ }^{1}$ Amy Callear, ${ }^{1}$ Rachel Truscon, ${ }^{1}$ Emileigh Johnson, ${ }^{1}$ Richard Evans, ${ }^{2}$ Latifa Bazzi, ${ }^{1}$ Caroline Cheng, ${ }^{1}$ Mark S. Thompson, ${ }^{3}$ Emily T. Martin, ${ }^{1}$ and Arnold S. Monto ${ }^{1}$

'University of Michigan School of Public Health, Department of Epidemiology, Ann Arbor, Michigan, USA; ${ }^{2}$ US Department of Veterans Affairs, Ann Arbor, Michigan, USA; and ${ }^{3}$ Centers for Disease Control and Prevention, Influenza Division, Atlanta, Georgia, USA

Background. The evidence that influenza vaccination programs regularly provide protection to unvaccinated individuals (ie, indirect effects) of a community is lacking. We sought to determine the direct, indirect, and total effects of influenza vaccine in the Household Influenza Vaccine Evaluation (HIVE) cohort.

Methods. Using longitudinal data from the HIVE cohort from 2010-11 through 2017-18, we estimated direct, indirect, and total influenza vaccine effectiveness (VE) and the incidence rate ratio of influenza virus infection using adjusted mixed-effect Poisson regression models. Total effectiveness was determined through comparison of vaccinated members of full or partially vaccinated households to unvaccinated individuals in completely unvaccinated households.

Results. The pooled, direct VE against any influenza was 30.2\% (14.0-43.4). Direct VE was higher for influenza A/H1N1 43.9\% (3.9 to 63.5 ) and B $46.7 \%$ (17.2 to 57.5) than $\mathrm{A} / \mathrm{H} 3 \mathrm{~N} 231.7 \%$ (10.5 to 47.8 ) and was higher for young children $42.4 \%$ (10.1 to 63.0) than adults $18.6 \%$ (-6.3 to 37.7). Influenza incidence was highest in completely unvaccinated households (10.6 per 100 personseasons) and lower at all other levels of household vaccination coverage. We found little evidence of indirect VE after adjusting for potential confounders. Total VE was $56.4 \%(30.1-72.9)$ in low coverage, $43.2 \%(19.5-59.9)$ in moderate coverage, and $33.0 \%(12.1$ to 49.0 ) in fully vaccinated households.

Conclusions. Influenza vaccines may have a benefit above and beyond the direct effect but that effect in this study was small. Although there may be exceptions, the goal of global vaccine recommendations should remain focused on provision of documented, direct protection to those vaccinated.

Keywords. influenza; vaccine effectiveness; cohort study; indirect protection.

Influenza vaccine is the best way to prevent influenza virus infections and their subsequent complications, including hospitalization and death. Despite a universal recommendation in the United States for annual influenza vaccination of all individuals $>6$ months old [1], uptake has been consistently suboptimal at approximately $45 \%$ [2]. Seasonal epidemics of influenza continue to cause substantial morbidity and mortality $[3,4]$ and the direct vaccine effectiveness (VE) in those vaccinated varies by season and dominant influenza subtype. On an annual basis, direct VE is most commonly estimated by test negative design

Received 23 February 2021; editorial decision 21 April 2021; published online 5 May 2021 Correspondence: R. Malosh, 1415 Washington Heights, M5134 SPH II, Ann Arbor, Ml 48109 (rmalosh@umich.edu).

Clinical Infectious Diseases ${ }^{\circledR} \quad$ 2021;73(7):1248-56

(C) The Author(s) 2021. Published by Oxford University Press for the Infectious Diseases Society of America. This is an Open Access article distributed under the terms of the Creative Commons Attribution-NonCommercial-NoDerivs licence (http://creativecommons.org/licenses/ by-nc-nd/4.0/), which permits non-commercial reproduction and distribution of the work, in any medium, provided the original work is not altered or transformed in any way, and that the work is properly cited. For commercial re-use, please contact journals.permissions@oup.com DOI: 10.1093/cid/ciab395 studies of medically attended illnesses, which does not account for the entire benefit of influenza vaccination, including protection against mild illness and potential indirect effects through herd immunity.

The term herd immunity describes a scenario under which population level immunity to an infection is sufficiently high that epidemics become less likely or start to diminish $[5,6]$. Vaccinated individuals have immunity from their vaccination and are thus protected from infection (direct VE). Unvaccinated individuals, who are not immune, receive indirect protection, primarily through reduction of the number of infectious individuals at the population level. The indirect effect is therefore distinct from the direct effect of the vaccine. The total effect of a vaccine represents the combination of direct and indirect effects [7].

A substantial amount of influenza virus transmission is thought to happen in settings with close and prolonged contact, such as households [8]. This setting is thus an ideal place to estimate indirect and total effects. There is some evidence of indirect effects from randomized studies, but these studies are limited by shorter follow-up or unique populations. A cluster randomized trial in 
Hutterite communities in Canada [9] showed substantial indirect effects of inactivated influenza vaccines, surprisingly nearly as high as direct effects when $80 \%$ of children were vaccinated [9, 10]. A modeling study informed by another randomized trial in Hong Kong estimated that, for influenza B, the indirect protection for household contacts could reach $20 \%$ under certain scenarios of household transmission and vaccination coverage [11]. Additional cluster-randomized studies in India [12] and Senegal [13,14] have estimated indirect and total VE, with mixed results.

The Household Influenza Vaccine Evaluation (HIVE) Cohort has evaluated influenza vaccine effectiveness longitudinally since 2010. The prospective design of the cohort and active surveillance for acute respiratory illness (ARI) presents a unique opportunity to observe both direct and indirect impacts of vaccination. Here we estimate the direct, indirect, and total vaccine effectiveness of influenza vaccine in households with children over 8 influenza seasons.

\section{METHODS}

\section{Study Population}

We used longitudinal data from the HIVE study collected from 2010-11 through 2017-18 influenza seasons. These data include active ARI surveillance from 3909 individuals from 911 distinct households, for a total of 9371 person-seasons. Recruitment and retention of participants has been previously described [15]. The HIVE study is approved by the institutional review board at the University of Michigan Medical School.

\section{Influenza Vaccination and Household Vaccination Coverage}

Influenza vaccination status was determined by a combination of self-report and documentation from electronic medical records (EMR) and the Michigan Care Improvement Registry (MCIR), as previously described [15-18]. Individuals were considered vaccinated $\geq 14$ days after their vaccination. We calculated the proportion of vaccinated household members and the incidence of influenza virus infection each season and longitudinally (ie, pooled over 8 years). We estimated the crude incidence rate of influenza in households by level of vaccination coverage (ie, completely unvaccinated, low vaccination coverage $[>0-50 \%]$, moderate vaccination coverage [51-99\%], and fully vaccinated). We calculated crude incidence rate, incidence rate ratios and $95 \%$ confidence intervals (CI) using the $\mathrm{R}$ package epitools.

\section{Influenza Infection Status}

Surveillance for acute respiratory illness was carried out from October through May (2010-11 through 2014-15 seasons) or year-round (2015-16 through 2017-18 seasons), as previously described [15]. Respiratory specimens were tested for influenza by RT-PCR, including A(H3N2), A(H1N1)pdm09 subtypes and $\mathrm{B}$ (Yamagata) and $\mathrm{B}$ (Victoria) lineages, using protocols from the Centers for Disease Control and Prevention.
Influenza Incidence and Direct Effect of Influenza Vaccination

We estimated the direct effects of influenza vaccination by comparing the seasonal incidence rate among vaccinated and unvaccinated individuals. Adjusted incidence rate ratios (aIRR) were estimated from mixed-effect Poisson regression models. $\mathrm{VE}_{\mathrm{D}}$ was calculated as 1 -aIRR ${ }^{\star} 100$. Vaccination was modeled as a time varying covariate, with some individuals contributing both vaccinated and unvaccinated person-time. Adjusted models included an offset term to account for person time and included potential confounders (age group, sex, and the Advisory Committee on Immunization Practices [ACIP] defined high-risk conditions [19]).

\section{Indirect and Total Vaccine Effectiveness: Mini-Community Design}

In this study we considered each household in the HIVE cohort to be a mini-community. The mini-community framework treats the household (or another small unit where contact is sufficient for transmission to occur) as the unit in which indirect and total effects of vaccination are to be estimated [20].

We fitted separate mixed-effects Poisson regression models to estimate $\mathrm{VE}_{\mathrm{I}}$ and $\mathrm{VE}_{\mathrm{T}}$, with random effects for household and season. Models were adjusted for potential confounders (age group, sex, and ACIP defined high-risk conditions). To estimate $\mathrm{VE}_{\mathrm{I}}$ we compared the incidence rate of influenza in unvaccinated individuals in completely unvaccinated households to unvaccinated members of households with higher levels of vaccination (ie, low [ $>0-50 \%$ ] or moderate [51-99\%] coverage). $\mathrm{VE}_{\mathrm{T}}$ was estimated by comparing the incidence rate of influenza in vaccinated individuals in completely and partially vaccinated households to unvaccinated individuals in completely unvaccinated households (Figure 1).

All statistical models were run in $\mathrm{R}$ software, version 4.0.2. Effect estimates were considered statistically significant if $95 \%$ confidence interval (CI) did not include the null value.

\section{RESULTS}

We followed 3416 individuals from 799 distinct households, for a total of 9371 person-seasons. Each household was followed for a median of 2 seasons (range 1-8, interquartile range [IQR] 1-4). The majority of the observed person-time was in children (58.8\%). School-aged children (5-17 years old) in particular, contributed 4184 (44.7\%) person-seasons of follow-up. No differences in age distribution were noted by household vaccination coverage. 1753 (48.7\%) individuals were female, contributing 4775 (51.0\%) person-seasons of follow-up. The HIVE cohort is predominantly White, and $16 \%$ of participants were considered high risk according to the ACIP definition (Table 1).

\section{Influenza Vaccination Coverage}

Approximately $65 \%$ of the HIVE cohort is vaccinated against influenza each year. In total, 6356 (68\%) person-seasons among vaccinated individuals over 8 seasons of follow-up 


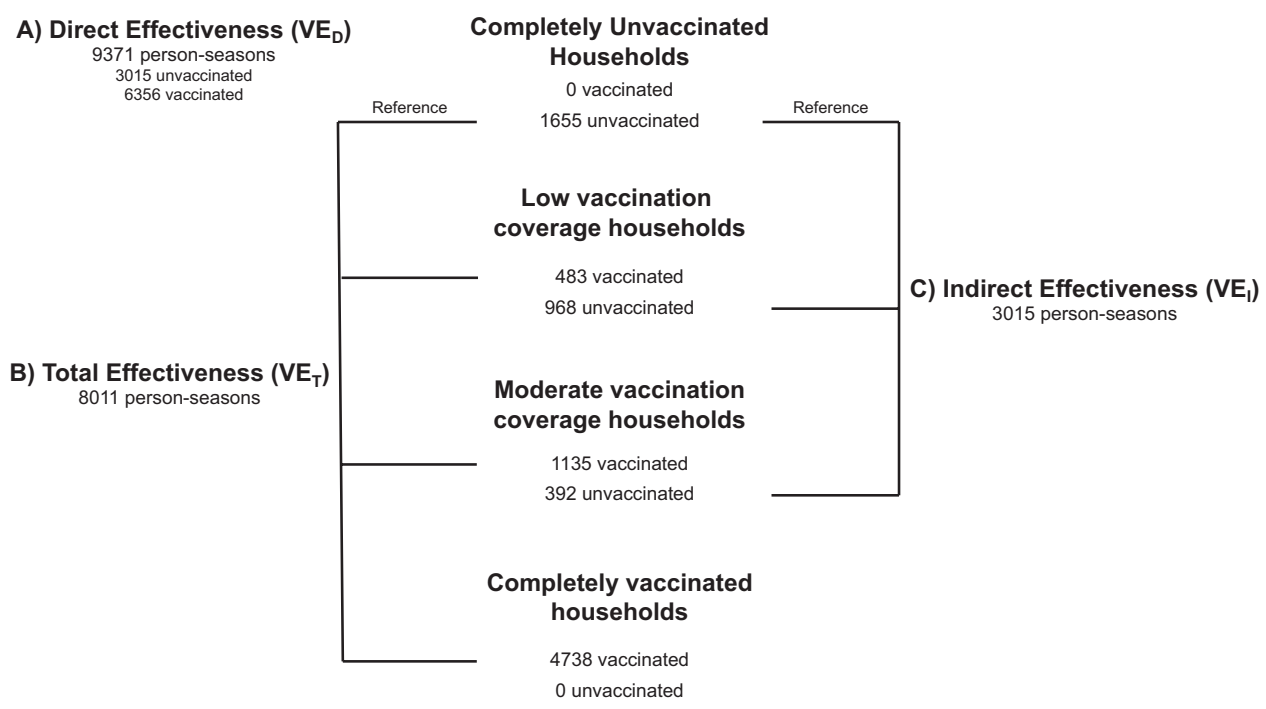

Figure 1. Person-seasons of follow-up for comparisons of influenza incidence. $A$, Direct effectiveness $\left(V_{D}\right)$ comparing vaccinated and unvaccinated individuals. $B$, Total effectiveness $\left(\mathrm{VE}_{\mathrm{T}}\right)$ comparing vaccinated members of moderate and low vaccination coverage households to unvaccinated members of completely unvaccinated households.

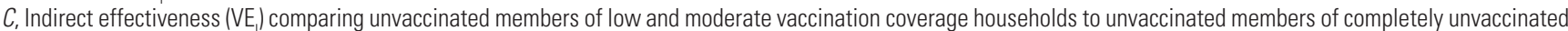
households, HIVE study, 2010-11 through 2017-18 seasons. Abbreviation: HIVE, Household Influenza Vaccine Evaluation.

were included in this analysis. At the household level, 52\% households were completely vaccinated each year, on average (Figure 2). The proportion of households that were completely vaccinated ranged from $47 \%$ in $2010-11$ to $60 \%$ in 2017-18. The percentage of households that were completely unvaccinated ranged from a high of $21 \%$ in $2010-11$ to a low of $11 \%$ in $2017-18$. On average, $17 \%$ of households were completely unvaccinated and $31 \%$ of households were partially vaccinated each season. There was little variability in vaccination coverage within households among children, pre-school aged children, or school-aged children. In most households, either all children were vaccinated, or none were (Figure S1).

\section{Influenza Incidence and Direct Effectiveness}

The incidence rate of influenza overall was 8.1 per 100 person-seasons (95\% CI 7.5-8.7; Table 2). Over the 8 seasons of follow-up incidence was highest for influenza A/ H3N2 infections (4.6 per 100 person-seasons, 95\% CI 4.2$5.1)$, followed by influenza $B$ (2.3 per 100 person-seasons, 95\% CI 2.0-2.6) and influenza A/H1N1 (1.1 per 100 personseasons, 95\% CI 0.9-1.4). Incidence rates for any influenza infection were higher in both preschool (0-4 years; 10.7 per 100 person-seasons, 95\% CI 9.0-12.6) and school-aged children (5-17 years; 9.1 per 100 person-seasons, 95\% CI 8.2-10.1), and lower among adults ( $\geq 18$ years; 6.1 per 100 person-seasons, 95\% CI 5.4-6.9). Seasonal incidence of any

Table 1. Baseline Demographics of Household Influenza Vaccine Evaluation (HIVE) Study Participants and Person-time Observed by Household Vaccination Coverage, 2010-11 Through 2017-18 Seasons

\begin{tabular}{|c|c|c|c|c|c|}
\hline & \multirow[b]{2}{*}{ N Individuals (\%) } & \multicolumn{4}{|c|}{ Person-Seasons of Follow-up N (\%) } \\
\hline & & Completely Unvaccinated & Low Vaccination Coverage & Moderate Vaccination Coverage & Fully Vaccinated \\
\hline Total & $3416(100)$ & 1655 & 1451 & 1527 & 4738 \\
\hline Female & $1753(49)$ & $855(49)$ & $737(49)$ & $755(51)$ & $2428(49)$ \\
\hline \multicolumn{6}{|l|}{ Age group, $y$} \\
\hline $0-5$ & $748(22)$ & $208(13)$ & $166(11)$ & $241(16)$ & $710(15)$ \\
\hline $5-17$ & $1251(37)$ & $813(49)$ & $686(47)$ & $645(42)$ & $2040(43)$ \\
\hline $18-49$ & $1310(38)$ & $588(36)$ & 539 (37) & $571(37)$ & $1760(37)$ \\
\hline$\geq 50$ & 107 (3) & $46(3)$ & $60(4)$ & $70(5)$ & $228(5)$ \\
\hline \multicolumn{6}{|l|}{ Race } \\
\hline White & $2496(73)$ & $1184(72)$ & $1073(74)$ & $1065(70)$ & $695(78)$ \\
\hline Black & $291(9)$ & $163(10)$ & $122(8)$ & $124(8)$ & $251(5)$ \\
\hline Asian & $279(8)$ & $85(5)$ & $93(7)$ & $177(12)$ & $444(9)$ \\
\hline Other & $350(10)$ & $169(10)$ & $115(8)$ & $144(10)$ & $303(6)$ \\
\hline High-risk condition & $537(16)$ & $192(12)$ & $252(17)$ & $270(18)$ & $807(17)$ \\
\hline
\end{tabular}




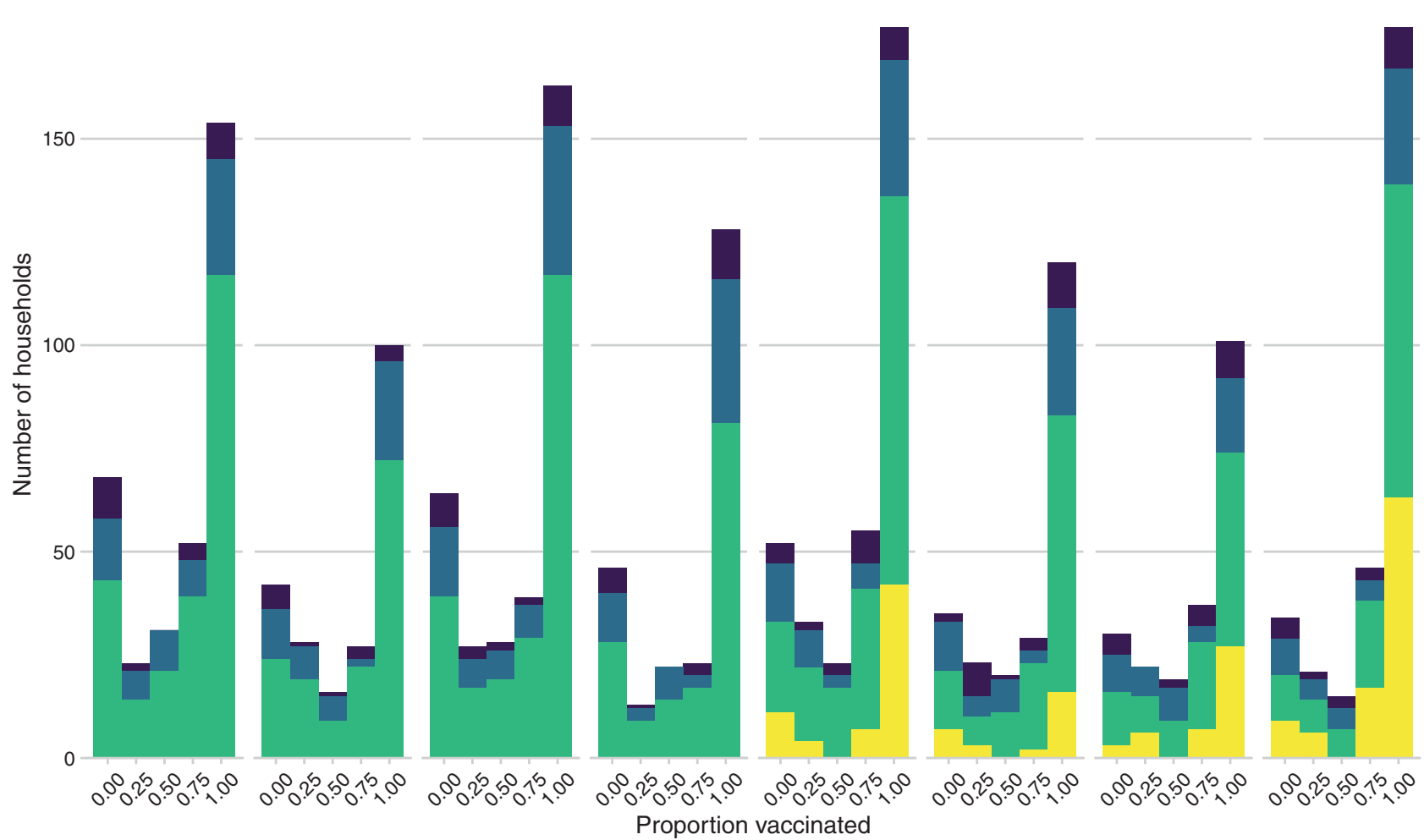

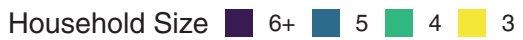

Figure 2. Distribution of household vaccination coverage by household size and season, 2010-11 through $2017-18$ seasons.

influenza virus infection (Table 2) was higher among unvaccinated individuals (10.0 per 100 person-seasons, 95\% CI 8.9-11.2) than vaccinated individuals (7.2 per 100 personseasons, 95\% CI 6.6-7.9). For influenza B, age stratified incidence rates (Supplementary Table 1) showed substantially higher incidence in preschool (3.1 per 100 person-seasons) and school-aged children (2.9 per 100 person-seasons) than among adults (1.4 per 100 person-seasons).

The overall adjusted $\mathrm{VE}_{\mathrm{D}}$ was $30.2 \%$ (95\% CI 14.0-43.4) with limited variation by type $40.7 \%$ (95\% CI 3.9-63.5); 31.7\% (95\% CI 10.5 to 47.8 ); $46.7 \%$ (95\% CI 17.2 to 57.5 ) for A/H1N1, A/ $\mathrm{H} 3 \mathrm{~N} 2$, and influenza $\mathrm{B}$, respectively). In age-group stratified models, we observed higher levels of protection for preschool (0-4 years; $\mathrm{VE}_{\mathrm{D}} 42.4 \%$, 95\% CI 10.1 to 63.0$)$ and school-aged children (5-17 years; $\mathrm{VE}_{\mathrm{D}} 28.7 \%$, 95\% CI 10.4 to 43.3 ) than we did for adults ( $\geq 18$ years; $\mathrm{VE}_{\mathrm{D}} 18.6,95 \% \mathrm{CI}-6.3$ to 37.7 ).

\section{Indirect Effectiveness of Influenza Vaccines}

Influenza incidence was generally highest among individuals in completely unvaccinated households (Figure 3). Overall, there appears to be a decline in influenza incidence with increasing proportion of household vaccinated. However, in some seasons, such as 2014-15, when the estimates of $\mathrm{VE}_{\mathrm{D}}$ in this cohort were essentially zero, the incidence rate was higher than in other seasons, and no decline was observed with increasing household vaccination coverage.

For indirect effect estimates we included a total of 3015 person-seasons of observation in unvaccinated individuals (Figure 1). We observed a lower incidence of influenza among unvaccinated individuals in moderately vaccinated households (8.9 infections per 100 person-seasons [95\% CI 6.2-12.4]) compared to those in completely unvaccinated households (10.6 infections per 100 person-seasons [95\% CI 9.1-12.3]). Point estimates for crude indirect vaccine effectiveness $\left(\mathrm{VE}_{\mathrm{I}}\right)$ comparing unvaccinated individuals in moderate vaccination coverage households to those in completely unvaccinated households suggest low levels of protection but were not statistically different from zero ( $\mathrm{VE}_{\mathrm{I}} 15.695 \% \mathrm{CI}-21.4$ to 41.3 ). The observed crude $\mathrm{VE}_{\mathrm{I}}$ in low vaccination coverage households was $2.4 \%$ (95\% CI -24.9 to 24.6 ; Table 3 ).

In both unadjusted and adjusted models there was no significant reduction in influenza incidence comparing unvaccinated individuals in low or moderately vaccinated households to those in completely unvaccinated households (Table 3). Age-group stratified models (Supplementary Table 2) demonstrate that school-aged children had lower indirect effect estimates in low ( $-3.4 \% 95 \%$ CI -52.9 to 30.0$)$ and in moderate ( $-69.8 \% 95 \%$ CI -216.9 to 9.0 ) coverage households than either pre-school aged 
Table 2. Seasonal Incidence Rate $(95 \% \mathrm{CI})$ of Influenza Infection and Direct Vaccine Effect (VED) Overall and Stratified by Type/Subtype and Age Group From the Household Influenza Vaccine Evaluation (HIVE) Study, Pooled Over 8 Seasons (2010-11 Through 2017-18 Seasons)

\begin{tabular}{|c|c|c|c|c|c|c|}
\hline & $\begin{array}{l}\text { Influenza } \\
\text { Infections }\end{array}$ & $\begin{array}{l}\text { Person- } \\
\text { seasons }\end{array}$ & $\begin{array}{l}\text { Incidence Rate per } 100 \\
\text { person-seasons (95\% Cl) }\end{array}$ & Crude VE ${ }_{D}{ }^{a}$ & $\begin{array}{l}\text { Unadjusted VE } E_{D} \text { With } \\
\text { Random Effects }\end{array}$ & $\begin{array}{l}\text { Adjusted VE } E_{D} \text { With } \\
\text { Random Effects }{ }^{c}\end{array}$ \\
\hline Any influenza & 760 & 9371 & $8.1(7.5-8.7)$ & & & \\
\hline Unvaccinated & 300 & 3007 & $10.0(8.9-11.2)$ & Ref & Ref & Ref \\
\hline Vaccinated & 460 & 6364 & 7.2 (6.6-7.9) & $27.5(16.2-37.4)$ & $32.6(19.8-43.7)$ & $30.2(14.0-43.4)$ \\
\hline \multicolumn{7}{|c|}{ Influenza type/subtype } \\
\hline Influenza A & 546 & 9371 & $5.8(5.3-6.3)$ & & & \\
\hline Unvaccinated & 209 & 3007 & $7.0(6.0-8.0)$ & Ref & Ref & Ref \\
\hline Vaccinated & 337 & 6364 & $5.3(4.7-5.9)$ & 23.8 (9.5-35.9) & $28.5(13.0-41.2)$ & $29.0(10.0-44.0)$ \\
\hline Influenza A/H3N2 & 431 & 9371 & $4.6(4.2-5.1)$ & & & \\
\hline Unvaccinated & 161 & 3007 & $5.4(4.6-6.2)$ & Ref & Ref & Ref \\
\hline Vaccinated & 270 & 6364 & $4.2(3.8-4.8)$ & $20.8(3.7-34.8)$ & $27.1(9.9-41.1)$ & 31.7 (10.5-47.8) \\
\hline Influenza A/H1N1 & 107 & 9371 & $1.1(0.9-1.4)$ & & & \\
\hline Unvaccinated & 48 & 3007 & $1.6(1.2-2.1)$ & Ref & Ref & Ref \\
\hline Vaccinated & 59 & 6364 & $0.9(0.7-1.2)$ & $41.9(15.0-60.3)$ & $38.7(0.4-62.3)$ & 40.7 (3.9-63.5) \\
\hline Influenza B & 216 & 9371 & $2.3(2.0-2.6)$ & & & \\
\hline Unvaccinated & 91 & 3007 & $3.0(2.4-3.7)$ & Ref & Ref & Ref \\
\hline Vaccinated & 125 & 6364 & $2.0(1.6-2.3)$ & $35.1(15.0-50.5)$ & $37.9(13.8-55.2)$ & $46.7(17.2-57.5)$ \\
\hline \multicolumn{7}{|l|}{ Age group } \\
\hline $0-4$ y & 142 & 1325 & $10.7(9.0-12.6)$ & & & \\
\hline Unvaccinated & 55 & 325 & $16.9(12.7-22.0)$ & Ref & Ref & Ref \\
\hline Vaccinated & 87 & 1000 & 8.7 (7.0-10.7) & $48.6(28.1-63.3)$ & $49.7(26.3-65.7)$ & $42.4(10.1-63.0)$ \\
\hline $5-17$ y & 382 & 4184 & $9.1(8.2-10.1)$ & & & \\
\hline Unvaccinated & 163 & 1433 & $11.4(9.7-13.3)$ & Ref & Ref & Ref \\
\hline Vaccinated & 219 & 2751 & $8.0(6.9-9.1)$ & $30.0(14.3-42.9)$ & 33.3 (16.3-46.8) & $28.7(10.4-43.3)$ \\
\hline$\geq 18 y$ & 236 & 3862 & $6.1(5.4-6.9)$ & & & \\
\hline Unvaccinated & 92 & 1257 & $7.3(5.9-9.0)$ & Ref & Ref & Ref \\
\hline Vaccinated & 144 & 2605 & $5.5(4.7-6.5)$ & $24.5(1.9-41.9)$ & $26.5(4.5-43.4)$ & $18.6(-6.3-37.7)$ \\
\hline $\begin{array}{l}\text { Abbreviations: } \mathrm{Cl} \text {, cor } \\
{ }^{a} \text { Direct vaccine effect } \\
{ }^{\mathrm{b}} \text { Mixed-effects Poissc }\end{array}$ & $\begin{array}{l}\text { rval; Ref, refer } \\
\text { ted as } 100 *(1 \\
\text { models with }\end{array}$ & effects for & old and season. & & & \\
\hline \multicolumn{7}{|c|}{$\begin{array}{l}\text { 'Mixed-effects Poisson regression models adjusted for age group, sex, calendar time, and high-risk condition. } \\
\text { dTwo influenza illnesses were influenza A/influenza B coinfections. These illnesses are considered as one infection for incidence rate calculations of any influenza and separately for type } \\
\text { specific incidence rate estimates. }\end{array}$} \\
\hline
\end{tabular}

children (low $\mathrm{VE}_{\mathrm{I}} 11.7 \% 95 \% \mathrm{CI}-63.8$ to 52.4 moderate $\mathrm{VE}_{\mathrm{I}}$ $10.2 \% 95 \% \mathrm{CI}-195.3$ to 72.7 ) or adults (low $\mathrm{VE}_{\mathrm{I}} 15.3 \% 95 \% \mathrm{CI}$ -38.3 to 48.2 ; moderate $\mathrm{VE}_{\mathrm{I}} 21.195 \% \mathrm{CI}-37.6$ to 54.8 ), suggesting that school-aged children were not as well protected by the vaccination status of their close contacts. Age-stratified $\mathrm{VE}_{\mathrm{I}}$ estimates had had broad and overlapping confidence intervals. We also estimated $\mathrm{VE}_{\mathrm{I}}$ by influenza type/subtype; $\mathrm{VE}_{\mathrm{I}}$ was lowest for influenza $\mathrm{A} / \mathrm{H} 3 \mathrm{~N} 2$ (low $\mathrm{VE}_{\mathrm{I}}-32.2 \% 95 \% \mathrm{CI}-94.4$ to 10.1); moderate $\mathrm{VE}_{\mathrm{I}}-19.7 \% 95 \% \mathrm{CI}-111.4$ to 32.2 ) and highest for influenza A/H1N1 low $\mathrm{VE}_{\mathrm{I}} 56.3 \% 95 \% \mathrm{CI}-9.2$ to 82.5 ; moderate $\mathrm{VE}_{\mathrm{I}} 24.9 \% 95 \% \mathrm{CI}-122.9$ to 74.7$)$, matching the $\mathrm{VE}_{\mathrm{D}}$ estimates (Supplementary Table 3).

\section{Total Household Effect of Influenza Vaccines}

The total effect of influenza vaccines compares vaccinated individuals in households with varying levels of vaccination coverage to unvaccinated individuals in completely unvaccinated households. To estimate total effect of influenza vaccine $\left(\mathrm{VE}_{\mathrm{T}}\right)$ we included 8011 person-seasons of follow-up in the analytical subset. The crude incidence rate was again highest among individuals in completely unvaccinated households (10.6 per 100 person-seasons, 95\% CI 9.1-12.3) (Table 4). Among vaccinated individuals in low (5.5 per 100 person-seasons, 95\% CI 3.68.1), moderate (6.8 per 100 person-seasons, $95 \%$ CI 5.3-8.5), and fully vaccinated (7.9 per 100 person-seasons, 95\% CI 7.18.7) households the incidence rate was lower. Notably, there is substantial overlap in the confidence intervals of the incidence rate estimates among vaccinated individuals in households with varying levels of vaccination coverage.

We found a significant total effect of influenza vaccines. In low vaccination coverage and moderate coverage households, $\mathrm{VE}_{\mathrm{T}}$ was $56.4 \%$ (95\% CI 30.1 to 72.9 ) and $43.2 \%$ (95\% CI 19.5 to 59.9), respectively, after adjusting for potential confounders. For individuals in fully vaccinated households, $\mathrm{VE}_{\mathrm{T}}$ was also significant ( $\mathrm{VE}_{\mathrm{T}} 33 \%$ [95\% CI 12.1 to 49.0]), but this estimate was similar to overall $\mathrm{VE}_{\mathrm{D}}$. 


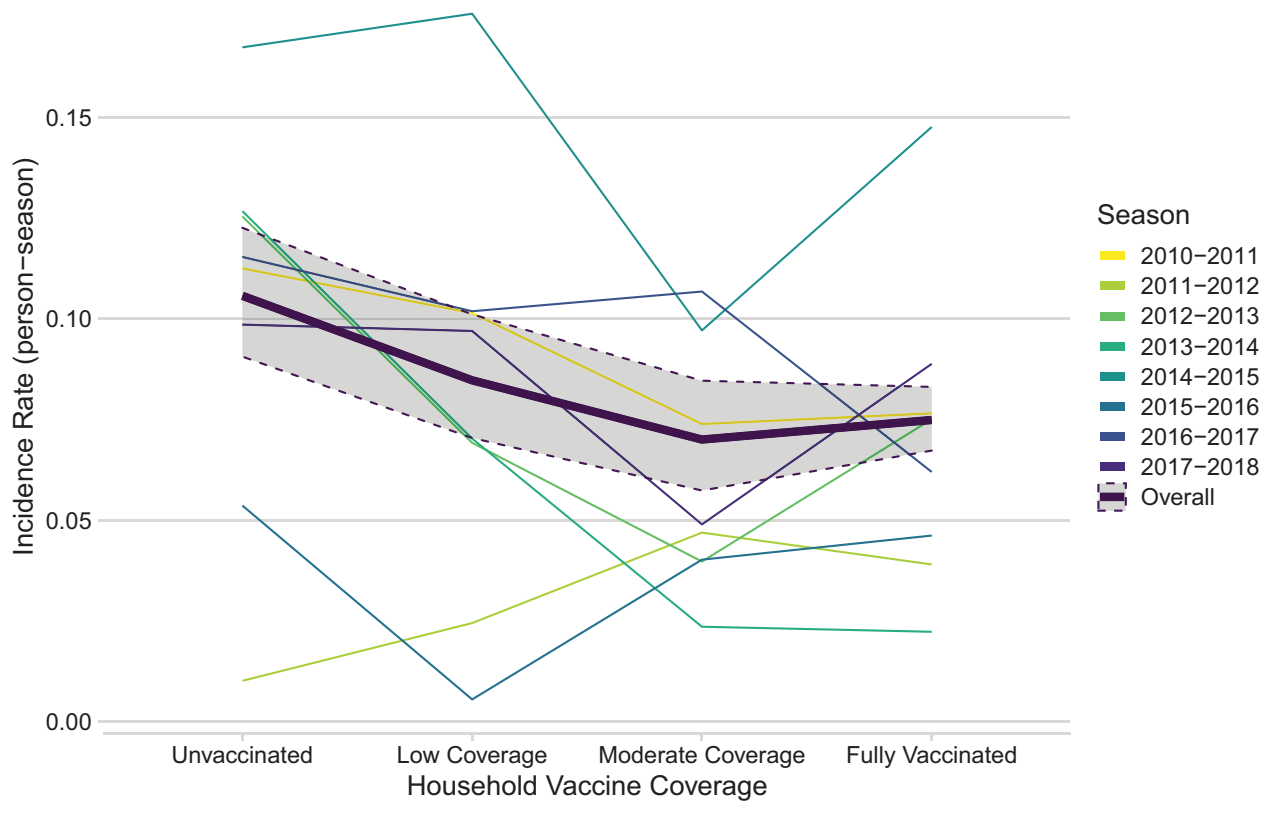

Figure 3. Incidence of influenza infection by household vaccination coverage and season, HIVE Study 2010-11 through 2017-18 seasons. Solid lines represent incidence rate estimates, dashed lines represent exact Poisson 95\% confidence intervals. Abbreviation: HIVE, Household Influenza Vaccine Evaluation.

\section{DISCUSSION}

In this prospective longitudinal study over 8 years, we were able to demonstrate nearly consistent moderate, direct protection against symptomatic influenza infection by vaccination. We also demonstrated significant total effectiveness that was statistically similar across households that varied in the extent of vaccination coverage. Indirect protection of unvaccinated people that live with vaccinated household contacts was not evident.

Direct protection of influenza vaccines has been demonstrated previously, but our findings include mild and moderate illnesses often missed by studies of medically attended acute respiratory illness (MAARI). This was particularly of interest for type B infections, which are more difficult to study with the MAARI design as longer duration of annual surveillance is needed to capture sufficient numbers of outcomes. In this study, direct influenza VE was highest for influenza $B$ and for $\mathrm{A} / \mathrm{H} 1 \mathrm{~N} 1$. The low estimates for $\mathrm{A} / \mathrm{H} 3 \mathrm{~N} 2$ reflect global patterns in vaccine effectiveness during this time period and are particularly driven by the 2014-15 season, when incidence was high, vaccine strain $(\mathrm{A} / \mathrm{Texas} / 50 / 2012)$ was considered a mismatch with predominant circulating viruses (genetic group 3c.2a), and vaccine effectiveness was near zero $[18,22,23]$.

In this study, the total effect of household vaccination was larger than the direct VE in low and moderate coverage households. Interestingly, we observed a trend of lower total VE point estimates with increasing household coverage. In fully vaccinated households, for example, total VE (33\%) was similar in magnitude to the direct VE (30\%), suggesting that protection in these households is primarily driven by the direct vaccine effects rather than a combination of direct and indirect effects. It is also possible that individuals in fully vaccinated households

Table 3. Seasonal Incidence Rate (95\% Cl) and Indirect Vaccine Effect (VEI) of Any Influenza Infection Among Unvaccinated Individuals by Household Vaccination Coverage in the Household Influenza Vaccine Evaluation (HIVE) Study population, Pooled Over 8 Seasons (2010-11 Through 2017-18 Seasons)

\begin{tabular}{|c|c|c|c|c|c|c|}
\hline & $\begin{array}{l}\text { Influenza } \\
\text { Infections }\end{array}$ & $\begin{array}{l}\text { Person-seasons of } \\
\text { Follow-up }\end{array}$ & $\begin{array}{c}\text { Seasonal Incidence Rate per } 100 \\
\text { person-seasons (95\% CI) }\end{array}$ & Crude VE ${ }_{1}^{a}$ & $\begin{array}{l}\text { Unadjusted VE, With } \\
\text { Random Effects }\end{array}$ & $\begin{array}{l}\text { Adjusted VE, With } \\
\text { Random Effects }\end{array}$ \\
\hline $\begin{array}{l}\text { Completely } \\
\text { unvaccinated }\end{array}$ & 175 & 1655 & $10.6(9.1-12.3)$ & Ref & Ref & Ref \\
\hline $\begin{array}{l}\text { Low vaccination } \\
\text { coverage }\end{array}$ & 100 & 968 & $10.3(8.4-12.6)$ & $2.4(-24.9$ to 24.6$)$ & 9.6 (-21.1 to 32.6$)$ & $8.2(-20.6$ to 30.1$)$ \\
\hline $\begin{array}{l}\text { Moderate } \\
\text { vaccination } \\
\text { coverage }\end{array}$ & 35 & 392 & $8.9(6.2-12.4)$ & $15.6(-21.4$ to 41.3$)$ & $16.9(-25.2$ to 44.9$)$ & $4.6(-41.2$ to 35.6$)$ \\
\hline
\end{tabular}

Abbreviations: $\mathrm{Cl}$, confidence interval; Ref, reference.

andirect vaccine effect (VE, calculated as 100*(1-alRR).

${ }^{b}$ Mixed-effects Poisson regression models with random effects for individual, household, and season.

${ }^{c}$ Mixed-effects Poisson regression models adjusted for age group, sex, calendar time, and high-risk condition. 
Table 4. Seasonal Incidence Rate and Total Vaccine Effect (VET) of Any Influenza Infection Comparing Vaccinated Individuals in Households With Varying Levels of Vaccination Coverage to Unvaccinated Individuals in Completely Unvaccinated Households, in the Household Influenza Vaccine Evaluation (HIVE) Study Population, Pooled Over 8 Seasons (2010-11Through 2017-18 Seasons)

\begin{tabular}{|c|c|c|c|c|c|c|}
\hline & $\begin{array}{l}\text { Influenza } \\
\text { Infections }\end{array}$ & $\begin{array}{l}\text { Person-seasons of } \\
\text { Follow-up }\end{array}$ & $\begin{array}{l}\text { Seasonal Incidence Rate per } 100 \\
\text { person-seasons }(95 \% \mathrm{Cl})\end{array}$ & Crude $V E_{T}{ }^{a}$ & $\begin{array}{l}\text { Unadjusted } \mathrm{VE}_{\mathrm{T}} \text { With } \\
\text { Random Effects }\end{array}$ & $\begin{array}{l}\text { Adjusted } V E_{T} \text { with } \\
\text { Random } \text { Effects }^{\circ}\end{array}$ \\
\hline $\begin{array}{l}\text { Completely } \\
\text { unvaccinated }\end{array}$ & 175 & 1655 & $10.6(9.1-12.3)$ & Ref & Ref & Ref \\
\hline $\begin{array}{l}\text { Low vaccination } \\
\text { coverage }\end{array}$ & 26 & 472 & $5.5(3.6-8.1)$ & 45.0 (16.9 to 64.7 ) & 57.7 (32.2-73.6) & $56.4(30.1-72.9)$ \\
\hline $\begin{array}{l}\text { Moderate } \\
\text { vaccination } \\
\text { coverage }\end{array}$ & 75 & 1109 & $6.8(5.3-8.5)$ & 33.5 (12.4 to 49.7$)$ & $41.5(17.0-58.7)$ & $43.2(19.5-59.9)$ \\
\hline Fully vaccinated & 379 & 4804 & $7.9(7.1-8.7)$ & 21.3 (5.5 to 44.5 ) & $33.0(12.1-49.0)$ & $33.0(12.1-49.0)$ \\
\hline
\end{tabular}

Abbreviations: $\mathrm{Cl}$, confidence interval; Ref, reference.

${ }^{a}$ Total vaccine effect $\left(V E_{1}\right)$ calculated as $100 *(1-a l R R)$.

${ }^{b}$ Mixed-effects Poisson regression models with random effects for individual, household, and season.

${ }^{c}$ Mixed-effects Poisson regression models adjusted for age group, sex, calendar time, and high-risk condition.

are more health-conscious and are more likely to report illnesses meeting our case definition and are thus more likely to have influenza detected. Our results are consistent with cluster randomized trials from India [12], which demonstrated substantial total VE, even in the absence of indirect protection. Similar patterns of total and indirect VE were also found in Senegal [13].

Studies of total and indirect protection of influenza vaccines have, in general, been limited to ecologic studies [24-27], modeling studies, or relatively small studies with non-specific outcomes (eg, febrile respiratory illness) [28, 29]. Few have been conducted in large populations in natural communities or in communities primarily vaccinated with inactivated influenza vaccine instead of live attenuated influenza vaccine. Previous individual-based studies have demonstrated indirect effects of influenza vaccine as large as the direct VE $[9,10]$, but the magnitude of indirect protection has varied based on the predominant circulating viruses. We expected lower indirect protection against influenza $\mathrm{A} / \mathrm{H} 3 \mathrm{~N} 2$ viruses for the same reason that we expected lower direct VE. Our findings (Supplementary Table 3) suggest that indirect protection, if present, may be higher for influenza $\mathrm{A} / \mathrm{H} 1 \mathrm{~N} 1$ but the small numbers in these stratified analyses limit our ability to draw conclusions.

In previous studies indirect effects have varied by age-group $[11,13]$. We explored the potential for effect modification using age-stratified models of indirect VE. We found that the lack of an observed indirect effect seems to be driven by the fact that schoolaged children did not benefit from vaccination of household contacts as adults and pre-school aged children did (Supplementary Table S2). This result is consistent with findings from Hong Kong showing that vaccinating children reduced infections in adult household contacts but did not reduce the overall infection probability [11]. School-aged children are known to drive influenza epidemics [30, 31], and as a result of their contact patterns [32], they represent the group at highest risk of community-acquired influenza. We did not collect data on contact patterns or schoollevel vaccination, limiting our ability to explore these effects. As shown by Tsang et al, the proportion of infections acquired in the household compared to the community has an impact on the overall indirect protection [11]. In addition, a number of recently observed issues affecting estimates of direct influenza VE may influence the indirect effects. Repeated annual vaccination, antigenic drift, mutations induced by growing vaccine viruses in chicken eggs, and waning immunity have all been linked to lower than expected direct VE in recent years [17, 18, 22, 33-37]. These mechanisms require further exploration, including individual hazard models to explore the relative contribution of community and household risk [38], as a potential explanation for the lack of indirect VE observed in this analysis.

Lack of heterogeneity in vaccine uptake is a challenge for evaluating indirect protection at the household level, as individuals share vaccination habits with others in their household. Also, most influenza infections in the HIVE study are considered community-acquired [16, 17], making identification of indirect protection resulting from reductions in household transmission risk a challenge. A more granular evaluation of indirect protection at the household level requires a situation where household members have similar risk of infection but different access to vaccination based on individual factors that are not shared by all household members. This is the situation that we are presented with given the US prioritization schemes for SARS-CoV-2 vaccine deployment. Although the household cohort was somewhat limited in the ability to evaluate potential indirect protection for unvaccinated members of partially vaccinated households, future findings in the era of COVID-19 may be quite different. Especially, as prioritization schemes will result in varied vaccination timing among household members. Similarly, the HIVE study population may not be generalizable to indirect effects observed in other settings (eg, high crowding, inadequate ventilation) with higher risk of household transmission.

We were unable to convincingly demonstrate indirect protection beyond the direct effect, but we did show that vaccine had a clear role in preventing mild disease in a cohort of families 
living in their own homes. The indirect effect might have been clear if the vaccines had higher direct effects. This is in agreement with recent literature reviews [39], meta-analyses [40], and cluster-randomized trials [12], which found inconsistency in demonstration of indirect effects. The entire concept of indirect protection or herd immunity has become a focus of efforts to control COVID-19 outbreaks. It is important to remember that the primary focus with a vaccine should be good direct VE and that indirect protection should be seen as a bonus, but not a necessity.

\section{Supplementary Data}

Supplementary materials are available at Clinical Infectious Diseases online. Consisting of data provided by the authors to benefit the reader, the posted materials are not copyedited and are the sole responsibility of the authors, so questions or comments should be addressed to the corresponding author.

\section{Notes}

Author Contributions. R. E. M., E. T. M., and A. S. M. conceptualized the data analysis. R. E. M., E. T. M., A. S. M., J. G. P., and M. S. T. contributed to the study design. J. G. P., A. C., R. E., L. B., and C. C. participated in data preparation and cleaning; R. T. and E. J. conducted laboratory assays; all authors participated in the writing and editing of the final manuscript.

Acknowledgments. The authors gratefully acknowledge the contribution of the HIVE study households. We also recognize the efforts of current and past HIVE Study staff Anne Kanicledes, Barbara Aaron, and Casey Martens; past study investigators Suzanne Ohmit; and current and past students Hannah Segaloff, Peter DeJonge, Kat Miller, and Khalil Chedid.

Disclaimer. The findings and conclusions in this report are those of the authors and do not necessarily represent the official position of the Centers for Disease Control and Prevention.

Financial support. This work was supported by the National Institute of Allergy and Infectious Diseases (NIAID) (grant number R03AI137658). The HIVE Study was further supported by the Centers for Disease Control and Prevention (CDC) (grant numbers U01 IP000170, U01 IP000474) and the NIAID (grant numbers R01 AI097150, R56 AI097150).

Potential Conflicts of Interest. J. P. reports grant K01 AI141579 outside the submitted work. E. M. reports grants from Merck, CDC, and National Institutes of Health (NIH) outside the submitted work; consulting fees from Pfizer; DSMB/Advisory board fees from NIH/NIAID Division of Microbiology and Infectious Diseases; Public Health Committee Advisory Group fees from Infectious Diseases Society of America (IDSA); and Annual Meeting Planning Committee fees from Society for Healthcare Epidemiology of America (SHEA), outside the submitted work. A. S. M. reports consulting fees from Sanofi-Pasteur and Seqirus, outside the submitted work. All other authors report no potential conflicts. All authors have submitted the ICMJE Form for Disclosure of Potential Conflicts of Interest. Conflicts that the editors consider relevant to the content of the manuscript have been disclosed.

\section{References}

1. Grohskopf LA, Sokolow LZ, Broder KR, Walter EB, Fry AM, Jernigan DB. Prevention and control of seasonal influenza with vaccines: recommendations of the advisory committee on immunization practices-United States, 2018-19 influenza season. MMWR Recomm Rep 2018; 67:1-20.

2. Centers for Disease Control and Prevention, National Center for Immunization and Respiratory Diseases (NCIRD). 2010-11 through 2017-18 Influenza Seasons Vaccination Coverage Trend Report | FluVaxView | Seasonal Influenza (Flu) | CDC. 2019 [cited 2019 Jun 14]. Available at: https://www.cdc.gov/flu/fluvaxview/ reportshtml/trends/index.html. Accessed 14 June 2019.

3. Rolfes MA, Foppa IM, Garg S, et al. Annual estimates of the burden of seasonal influenza in the United States: a tool for strengthening influenza surveillance and preparedness. Influenza Other Respir Viruses 2018; 12:132-7.
4. Reed C, Chaves SS, Daily Kirley P, et al. Estimating influenza disease burden from population-based surveillance data in the United States. PloS One 2015; 10. Available at: https://www.ncbi.nlm.nih.gov/pmc/articles/PMC4349859/.

5. Fox JP, Elveback LR. Chapter 16: herd immunity - changing concepts. In: Notkins AL, ed. Viral immunology and immunopathology. New York: Academic Press, 1975:273-90. Available at: http://www.sciencedirect.com/science/article/pii/B9780125220507500204.

6. Anderson RM, May RM. Vaccination and herd immunity to infectious diseases. Nature 1985; 318:323-9.

7. Halloran ME, Struchiner CJ, Longini IM Jr. Study designs for evaluating different efficacy and effectiveness aspects of vaccines. Am J Epidemiol 1997; 146:789-803.

8. Tsang TK, Lau LLH, Cauchemez S, Cowling BJ. Household transmission of influenza virus. Trends Microbiol 2016; 24 . Available at: http://www.cell.com/article/ S0966842X15002516/abstract.

9. Loeb M, Russell ML, Moss L, et al. Effect of influenza vaccination of children on infection rates in Hutterite communities: a randomized trial. JAMA 2010; 303:943-50.

10. Wang B, Russell ML, Moss L, et al. Effect of influenza vaccination of children on infection rate in Hutterite communities: follow-up study of a randomized trial. PLoS One 2016; $11: \mathrm{e} 0167281$.

11. Tsang TK, Fang VJ, Ip DKM, et al. Indirect protection from vaccinating children against influenza in households. Nat Commun 2019; 10:106.

12. Sullender WM, Fowler KB, Gupta V, et al. Efficacy of inactivated trivalent influenza vaccine in rural India: a 3-year cluster-randomised controlled trial. Lancet Glob Health 2019; 7:e940-50.

13. Niang MN, Sugimoto JD, Diallo A, et al. Estimates of inactivated influenza vaccine effectiveness among children in Senegal: results from 2 consecutive clusterrandomized controlled trials in 2010 and 2011. Clin Infect Dis 2021; 72:e959-69.

14. Diallo A, Diop OM, Diop D, et al. Effectiveness of seasonal influenza vaccination in children in Senegal during a year of vaccine mismatch: a cluster-randomized trial. Clin Infect Dis 2019; 69:1780-8.

15. Monto AS, Malosh RE, Evans R, et al. Data resource profile: Household Influenza Vaccine Evaluation (HIVE) Study. Int J Epidemiol Available at: http://academic. oup.com/ije/advance-article/doi/10.1093/ije/dyz086/5481893

16. Ohmit SE, Petrie JG, Malosh RE, et al. Influenza vaccine effectiveness in the community and the household. Clin Infect Dis 2013; 56:1363-9.

17. Ohmit SE, Petrie JG, Malosh RE, Fry AM, Thompson MG, Monto AS. Influenza vaccine effectiveness in households with children during the 2012-2013 season: assessments of prior vaccination and serologic susceptibility. J Infect Dis 2015; 211:1519-28.

18. Petrie JG, Malosh RE, Cheng CK, et al. The household influenza vaccine effectiveness study: lack of antibody response and protection following receipt of 2014-2015 influenza vaccine. Clin Infect Dis 2017; 65:1644-51.

19. Grohskopf LA. Prevention and control of seasonal influenza with vaccines: recommendations of the advisory committee on immunization practices - United States, 2020-21 influenza season. MMWR Recomm Rep. 2020; 69. Available at: https://www.cdc.gov/mmwr/volumes/69/rr/rr6908a1.htm.

20. Halloran ME. The minicommunity design to assess indirect effects of vaccination. Epidemiol Methods 2012; 1:83-105.

21. Rolfes MA, Flannery B, Chung JR, et al. Effects of influenza vaccination in the United States During the 2017-2018 influenza season. Clin Infect Dis 2019; 69:1845-53. Available at: http://academic.oup.com/cid/advance-article/ doi/10.1093/cid/ciz075/5305915.

22. Zimmerman RK, Nowalk MP, Chung J, et al. 2014-2015 Influenza vaccine effectiveness in the United States by vaccine type. Clin Infect Dis 2016; 63:1564-73.

23. Flannery B, Zimmerman RK, Gubareva LV, et al. Enhanced genetic characterization of influenza $\mathrm{A}(\mathrm{H} 3 \mathrm{~N} 2)$ viruses and vaccine effectiveness by genetic group, 2014-2015. J Infect Dis 2016; 214:1010-19.

24. Monto AS, Davenport FM, Napier JA, Francis T Jr. Modification of an outbreak of influenza in Tecumseh, Michigan, by vaccination of schoolchildren. J Infect Dis 1970; $122: 16-25$.

25. Glezen WP, Gaglani MJ, Kozinetz CA, Piedra PA. Direct and indirect effectiveness of influenza vaccination delivered to children at school preceding an epidemic caused by 3 new influenza virus variants. J Infect Dis 2010; 202:1626-33.

26. Piedra PA, Gaglani MJ, Kozinetz CA, et al. Trivalent live attenuated intranasal influenza vaccine administered during the 2003-2004 influenza type A (H3N2) outbreak provided immediate, direct, and indirect protection in children. Pediatrics 2007; 120:e553-564.

27. Gaglani MJ, Piedra PA, Herschler GB, et al. Direct and total effectiveness of the intranasal, live-attenuated, trivalent cold-adapted influenza virus vaccine against the 2000-2001 influenza $\mathrm{A}(\mathrm{H} 1 \mathrm{~N} 1)$ and B epidemic in healthy children. Arch Pediatr Adolesc Med 2004; 158:65-73.

28. Hurwitz ES, Haber M, Chang A, et al. Effectiveness of influenza vaccination of day care children in reducing influenza-related morbidity among household contacts. JAMA 2000; 284:1677-82. 
29. Esposito S, Marchisio P, Cavagna R, et al. Effectiveness of influenza vaccination of children with recurrent respiratory tract infections in reducing respiratoryrelated morbidity within the households. Vaccine $2003 ; 21: 3162-8$.

30. Worby CJ, Chaves SS, Wallinga J, Lipsitch M, Finelli L, Goldstein E. On the relative role of different age groups in influenza epidemics. Epidemics 2015; 13:10-6.

31. Heikkinen T. Influenza in children. Acta Paediatr 2006; 95:778-84.

32. Eames KT, Tilston NL, Brooks-Pollock E, Edmunds WJ. Measured dynamic social contact patterns explain the spread of H1N1v influenza. PLoS Comput Biol 2012 8:e1002425.

33. McLean HQ, Thompson MG, Sundaram ME, et al. Impact of repeated vaccination on vaccine effectiveness against influenza $\mathrm{A}(\mathrm{H} 3 \mathrm{~N} 2)$ and $\mathrm{B}$ during 8 seasons. Clin Infect Dis 2014; 59:1375-85.

34. Smith DJ, Forrest S, Ackley DH, Perelson AS. Variable efficacy of repeated annual influenza vaccination. Proc Natl Acad Sci U S A 1999; 96:14001-6.

35. Gouma S, Zost SJ, Parkhouse K, et al. Comparison of human H3N2 antibody responses elicited by egg-based, cell-based, and recombinant protein-based influenza vaccines during the 2017-2018 season. Clin Infect Dis 2019; 71:1447-53.

36. Ferdinands JM, Fry AM, Reynolds S, et al. Intraseason waning of influenza vaccine protection: evidence from the US influenza vaccine effectiveness Network, 2011-12 through 2014-15. Clin Infect Dis 2017; 64:544-50.

37. Cobey S, Gouma S, Parkhouse K, et al. Poor immunogenicity, not vaccine strain egg adaptation, may explain the low $\mathrm{H} 3 \mathrm{~N} 2$ influenza vaccine effectiveness in 2012-13. Clin Infect Dis 2018; 67:327-33.

38. Petrie JG, Eisenberg MC, Ng S, et al. Application of an individual-based transmission hazard model for estimation of influenza vaccine effectiveness in a household cohort. Am J Epidemiol 2017; 186:1380-8.

39. Mertz D, Fadel SA, Lam P-P, et al. Herd effect from influenza vaccination in nonhealthcare settings: a systematic review of randomised controlled trials and observational studies. Eurosurveillance 2016; 21:30378.

40. Arinaminpathy N, Kim IK, Gargiullo P, et al. Estimating direct and indirect protective effect of influenza vaccination in the United States. Am J Epidemiol 2017; 186:92-100. 